\title{
Fluorescence Angiography
}

National Cancer Institute

\section{Source}

National Cancer Institute. Fluorescence Angiography. NCI Thesaurus. Code C116569.

An examination technique that uses fluorescent dye and a specialized camera to inspect blood flow in the retina and choroid. 